\title{
Sciendo
}

\section{Data Mining Applications in SMEs: An Italian Perspective}

\author{
Amir Topalović, Antonia Azzini \\ Consortium for the Technology Transfer, C2T
}

\section{Abstract}

Background: From the last decade, data mining techniques, employed in particular in customer relationship management, have assumed a key role in the profitability and operations of companies. To support small and medium companies (SMEs), several innovative and continuously improving tools have been developed that allow SMEs to utilize the internal and external data sources to increase their competitiveness. Objectives: In this paper, an analysis of the impact of digitalization, and in particular data mining techniques, in the context of SMEs development is presented. Methods/Approach: A review of various sources has been conducted, with the focus on open source tools, since in the context of the Italian economy they are used by SMEs the most. Results: First, the analysis presents a brief review of the data mining techniques available and shows how they are practically employed in small companies. Second, an economical review of investments in data mining projects in Italy is presented. Conclusions: The review indicates that data mining techniques can boost a company in the market. However, the awareness of data mining as a company asset is still not strong in Italian SMEs and most investments in Italy are still carried out by large companies.

Keywords: SMEs, data mining, customer relationship management, information technology, knowledge management, big data, Internet of things.

JEL classification: $\mathrm{O} 32$

Paper type: Research article

Received: Apr 27, 2020

Accepted: Jul 07, 2020

Citation: Topalovic, A., Azzini, A., (2020), "Data Mining Applications in SMEs: An Italian Perspective", Business Systems Research, Vol 11 No 3, pp. 127-146.

DOI: https://doi.org/10.2478/bsrj-2020-0031

\section{Introduction}

From the last decades until today, customer relationship management has increasingly assumed a key role in the profitability and operations of companies, thanks to the management of the relationships with the market to which they are addressed (Ghaderi \& Fei, 2013). As widely reported in the literature (Ngai et al, 2009), the objective of Customer Relationship Management (CRM) has been based on the study and identification of the needs of the market to which a company turns. The aim is leveraging these aspects to increase the company's profitability in the long term 
(Stringfellow et al., 2004), clearly taking into account several environmental, social, economic, and financial factors that surround a company, but which are constantly evolving. Companies are required to implement solutions to be able to deal with these changes (Rygielski et al., 2002). In this context, the advent of information technology and in particular the introduction of innovative Knowledge Management techniques, and data mining more specifically, has transformed customer relationship management, as well as the way companies, manage information about their customers (Shaw et al., 2001).

Moreover, digital innovation increasingly present in recent years has strongly influenced and is still bringing, a continuous change in the social and economic logic (Pejić Bach, 2014, Ministry of Economic Development, 2017). This innovation also calls for a definition of new personal attitudes, as well as continuous updates visible in the business processes, in the individual company or enterprise, and increasingly greater contexts and territorial aggregations. It is possible to observe how these changes are directly affecting the dynamics of the information and communication technology market, which continues its rapid transformation into the direction already taken, highlighting at the same time the slowdown of more traditional technologies on the one hand and the dragging effect of new ones on the other. New strategies implemented in various contexts force companies to renew continually, with particular attention to all the new information and communication technology investments that the company must continuously employ.

The concept of a data-centric business model, which has been subject to discussion in the literature and the market for several years (Blagojevic \& Micić, 2013; Calvello et al., 2020), is becoming increasingly interesting and is therefore applied by SMEs, particularly in all economic sectors where new competitive scenarios arise. All this happens with particular attention to investments in information technology. Indeed, technologies and solutions involving processes such as data and information management and exploitation are among the most dynamic sectors in today's market.

Nowadays, popular technologies include machine learning, big data, and Analytic technologies, along with architectural and cloud-ready infrastructure technologies. These approaches have as a point of innovation the ability to reorganize completely how data is acquired and managed by end-users. One example is represented by machine learning and big data technologies, actually widely used to make real-time decisions and learn old and new processes. The combination of these new analytical and cognitive technologies is increasingly becoming what differentiates market leaders. In fact, in such a scenario what no company can avoid is finding new ways to achieve the goal to effectively predict and design new solutions to improve industrial processes, to assist people in the triple role of customers, citizens, patients, and finally, to make decisions in different market environments, currently dominated by systemic uncertainty and recurring turbulence.

According to the work carried out by Almeida and Bernardino (2016), it is difficult for SMEs to obtain important positions, with good profits, in the market, especially the global market. The growth of the global market causes new problems for companies. Extracting knowledge from the huge amount of data available to a company (whether it is data generated inside or outside) has been recognized as one of the most valuable ways to obtain a competitive advantage. In this sense, the recognition of the importance of data mining allowed this research area to be among the fields of computer science that has produced the biggest amount of research and development in recent years. The authors show how there are many use cases for data mining Tools, even though the most common consists of extracting patterns and 
trends stored in data and use these patterns to discover previously unknown information to be used in strategic management decisions (Shaw et al., 2001). This information comes in many forms and has a lot of characteristics that differentiate it from one another. To support SMEs to cope with the continuous evolution of the data mining tools used in a data management cycle, several innovative and continuously improving tools have been developed.

The goal of this paper is to present an analysis of the impact of digitalization, and in particular, data mining techniques in the context of customer relationship management applied to boost SMEs development. In detail, we aim to analyse only open source tools because they are the most used by SMEs. The analysis also presents a brief review of the data mining techniques available and shows how they are practically employed in small companies, with particular attention to the Italian situation.

We choose to analyse only open source tools because they require no acquisition costs, have bigger flexibility and a faster renovation due to their extensive development community, and are robust enough for SME's needs. The analysis presents a brief review of the data mining techniques available and shows how they are practically employed in small companies. Then we also present an economical review of investments in data mining projects in Italy, which on one side outlines that, once used, data mining techniques can boost a company in the market, on the other side the awareness of data mining as a company asset is still not strong in Italian SMEs and most investments in Italy are still carried out by biggest companies (Cedrola et al., 2009).

The remainder of this paper is structured as follows: after a background of the techniques already presented in the literature regarding the information and communication technology market, the basic concepts of the data mining approaches are summarized into Section 3. Section 4 reports the applications of such data mining solutions into SME applications, by introducing the advantages coming from the use of Customer Relationship Management Services in improving these applications. This Section also provides a detailed discussion of the Italian market. The next Section 5 focuses the attention on a set of techniques that are used, alone, or combined with the Data Mining, in the information and communication technology area for the SMEs. A snapshot of the Italian situation is summarized in Section 6, while Section 7 concludes with some final remarks.

\section{Literature Review}

\section{Data mining as a support to competitive advantage}

Digital innovation is bringing a continuous change in the socio-economic logics that affect the processes of both SMEs and large companies (Sali, 2020), in contexts ranging from territorial realities to the international situation.

In this context, the information and communication technology market is a good marker of the economic trend, both national and international, highlighting in general a slowdown in traditional technologies on the one hand, and a continuous increase in new paradigms on the other, primarily the so-called innovation accelerators. Innovative technologies and services, of primary interest to businesses, embrace different processes and technological solutions ranging from data and information management and exploitation, including machine learning, big data, and Analytics solutions, to processes based on new architectures and cloud infrastructures, as well as processes related to the Internet of Things and the management of e-commerce services. The different contributions reported by the literature (Assintel, 2019; Ministry of 
Economic Development, 2017; Rojas-Torre \& Kshetri, 2019) highlight the main aspects considered in the definition and application of innovative solutions to different markets, according to specific situations and contexts.

labal et al. (2018) present a qualitative research work regarding the use of big data to improve the overall management in SMEs, while an interesting comparative work related to the main data mining techniques applied for SME manufacturing is presented in (Packianather et al., 2017), and a survey on open-source data mining tools is reported by Almeida and Bernardino (2016).

A contribution showing the impact of the transformation from the more traditional use of information and communication technology to internet of things solutions is presented in (Abazi, 2016), where the authors explain in detail the influence of smart solutions to SMEs, while Jun et al. (2017) present a platform definition and integration to support smart solutions for SMEs. Moreover, another interesting approach highlights the versatility of the internet of things techniques, by describing a combined application of them with big data solutions for manufacturing industries (Mourtzis et al., 2016). In this topic, another interesting overview of big data for growth in SMEs is reported by Sen et al. (2016).

Another detailed work discussing the evolution of knowledge management into SMEs is reported by Alavi and Leidner (2001), in which three research questions have been identified, concerning the barriers hindering the spread of knowledge management practices in SMEs, the main Knowledge Management Systems (KMSs) adopted by SMEs, and the impact of the use of knowledge management practices on SME performance. In another work (Ghaderi \& Fei, 2013), the proposed knowledge management application highlights two groups of SMEs: in the first one the business is for the most part capital-based, and it belongs to the capital intensive organizations; in the second one the business is, for the most part, knowledge-based, then belonging to knowledge-intensive organizations. Finally, a recent comparative analysis is available (Alavi \& Leidner, 2001), here an empirical study has been carried out by comparing the international economical situation w.r.t. the improvement carried out by knowledge management activities. The qualitative side of this research is focused on observing existing SMEs and considering the big data literature (Landset et al., 2015; Sen et al., 2016) to offer in future research a new management system and innovative techniques.

As previously introduced and reported in the literature (Ngai et al., 2009), the information available to clients together with the information technology tools suitable for their management represents the basis on which the company's success strategies are mainly defined. With this trend, technologies such as data mining have opened a new era for customer relationship management, through which companies can gain competitive advantages. Data mining techniques are considered a leading customer relationship management tool that has been deeply influenced by advancements, more general in information technology applications, and in particular in the Digital Innovation area.

Until the last decade, much research has been carried out in the context of data mining in large companies (Hsu, 2009), while similar literature in SMEs has been very limited (Almeida \& Berbardino, 2016; Linoff et al., 2004). The reason was mainly due to the lack of investments by the companies themselves, as well as the limited availability of information to access.

The ever-increasing growth of Digital Innovation technologies for companies has led them to move from product-oriented business strategies to those oriented towards customer relations (Marzo \& Scarpino, 2016), with particular attention paid to service 
improvement activities that lead to customer loyalty in different segments (Yang et al., 2011).

In recent years, not only large companies and multinationals but also SMEs have been exploiting customer relationship management technologies to increase longterm profitability. Several authors claim that customer retention is important for SMEs to cope with the limited availability of resources, such as (Chen \& Popovich, 2003). On the other hand, Skaates and Seppänen (2002) also stress the importance of the role that customer relationship management activities acquire by contributing to the development of SMEs' competencies, while other authors stress precise customer segmentation and customer profiling and targeting (Gurău et al., 2003).

Despite the importance of data mining techniques to CRM, there is a dearth of literature in this area. According to the literature (Chen \& Popovich, 2003; Ghaderi \& Fei, 2013,), the implementation of data mining applications in customer relationship management follows an emerging trend among business organizations. Appropriate data mining tools, which are good at extracting and identifying useful information and knowledge from enormous customer databases, are one of the best supporting tools for making customer relationship management decisions (Berson et al., 1999). Consequently, the application of data mining techniques in CRM is worth pursuing in a customer-centric economy (Ngai et al., 2009).

The choice of the use of data mining Techniques in the definition and management of significant relationships and rules for the management of large amounts of data in a DB is supported in the literature by the increasing need of companies to define solutions able to extract and manage hidden knowledge within such data (Hui \& Jha, 2000; Marbán et al., 2008; Witten et al., 1999).

The implementation of data mining applications to CRM follows an emerging trend among corporate organizations. In particular, appropriate data mining tools, able to extract and identify useful information and knowledge from huge customer databases, have become one of the best decision support tools in CRM techniques (Berson et al., 1999). Increasingly, national and global economies are paying more and more attention to the customers to whom they are addressed, putting in the foreground the desires and needs of the various customers. Ngai et al. (2009) present an interesting discussion on the application of data mining techniques in CRM.

\section{An overview of data mining techniques}

Starting from a general definition, data mining is the discovery process of relationships, patterns, and previously unknown and potentially useful information within large databases (Ali \& Wasimi, 2009). A pattern indicates a structure, or, in general, a synthetic representation of data. Data mining can be defined as an analytical process, aimed at selecting and exploring models based on large quantities of data in the search for reports and information, in some cases unknown beforehand. This information can also be converted into commercial actions to obtain a business advantage.

In companies, data mining is often used to develop applications integrated into business decision-making processes, rather than studies of a single event or aspect, economic or business. Moreover, it allows for the integration of quantitative analysis and business knowledge. The schema reported in Figure 1 shows the rule assumed by data mining into a knowledge discovery from data process, together with the well known related functionalities (Fayyad et al., 1996; Luo, 2008). 
Figure 1

Functionalities of a knowledge discovery from data process

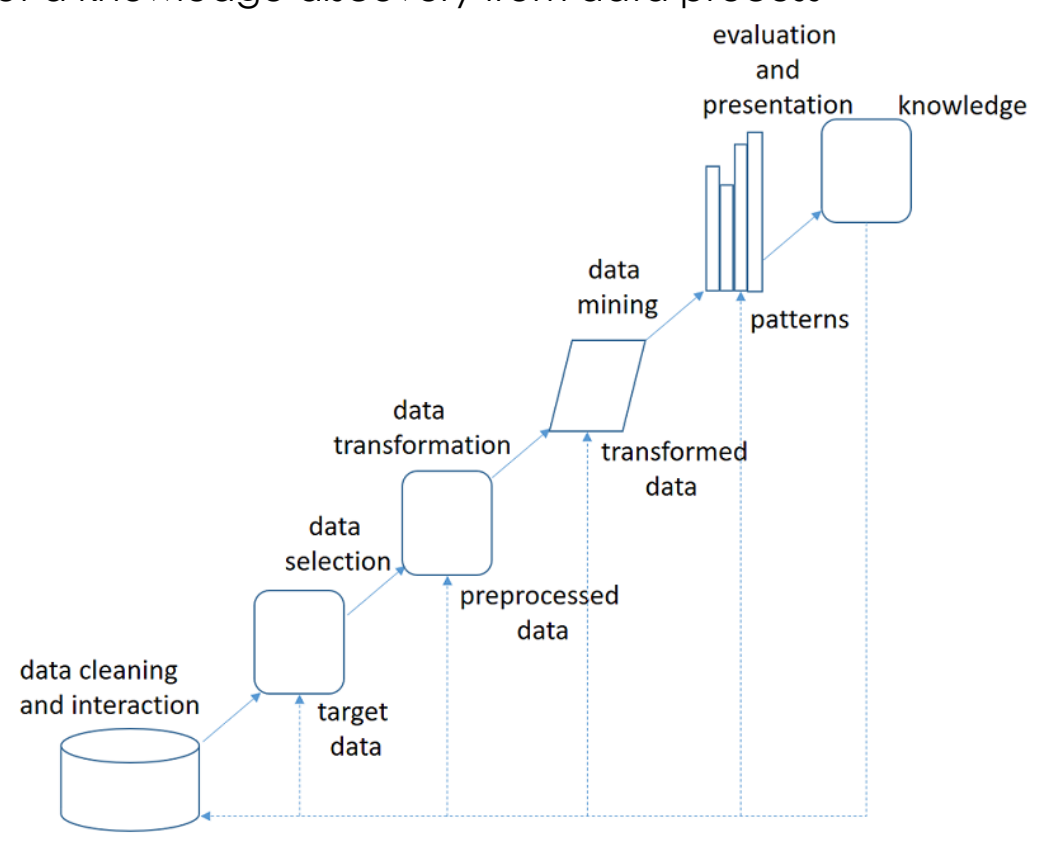

Source: Author's work (2020).

The multidisciplinary nature of data mining also allows the interaction between different technological solutions, including knowledge discovery techniques, extraction of information from Databases, statistical analysis techniques, as well as knowledge-based or self-learning systems. Indeed, statistical techniques help to check hypotheses using small random samples and sample sizes, whereas data mining automatically generates new hypotheses using an endless quantity of data. Artificial intelligence and machine learning are also used to analyse data.

Data mining presents several advantages, which can be summarized as follows: the processing of quantitative, qualitative, textual, image and sound data; a priori assumptions are not required; assumptions about the distributive form of the variables are not required; the processing of a large number of observations and variables; the implementation of optimized algorithms to minimize processing time; the simplicity of interpretation of the result; and the easy visualization of results.

The novelty offered by data mining tools is therefore based on the possibility of integrating (in companies where it is possible) decision-making processes with constructed rules, synthesizing high information assets (Ali \& Wasimi, 2009). The results obtained with data mining tools must, therefore, be presented, communicated, and shared with the company areas to obtain high benefits, thus defining reticular collaborative models.

The main methodologies that can be implemented through data mining techniques (Calvello, 2020) are summarized in the following points: (i) exploration through multidimensional visualization (multidimensional scaling, logistic regression analysis, stepwise, match analysis); (ii) association \& sequences (usually used in market basket analysis to measure product affinity); (iii) clustering activities (segmentation of customers into homogeneous groups); (iv) factorial analysis (to determine the number of factors to be extracted and for main components); ( $v$ ) prediction models based on classification algorithms (e.g. decision trees and neural networks); (vi) learning 
methods (e.g. kohonen maps and other unsupervised neural networks); and (vii) genetic algorithms.

Data mining techniques are oriented to business needs. Nowadays many processes in business are based on data. In such a context, Data mining helps to recognize trends and patterns to improve processes, develop business, and enjoy more success (Wanghualin, 2010). Examples of data mining applications are also represented in Figure 2.

Figure 2

Examples of data mining applications

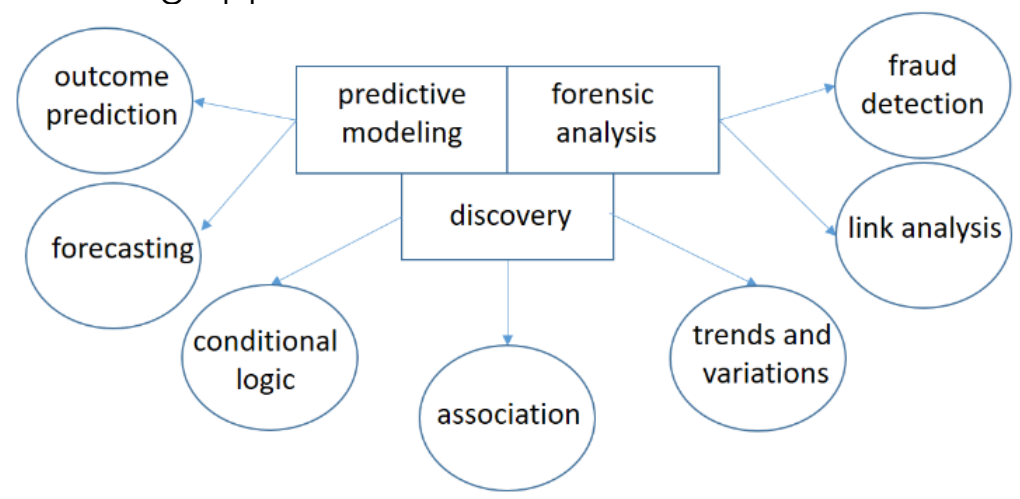

Source: Author's work (2020).

Enhancing the management of business information residing in large databases is one of the best-known objectives of data mining. However, the potential of the techniques, methodologies, and examples that are part of these methodologies go beyond simple data enhancement. Examples can be identified in:

- Clustering analysis, to identify target groups: in companies and in particular in business and marketing activities it is useful to segment (to the cluster) the database and provides a specific product or service to a given customers' selection. The different combinations of variables make the cluster analysis more or less selective according to the needs.

- Regression analysis is used for example in predictive marketing, where studies are conducted on changes, habits, customer satisfaction level, and other factors related to economic and financial parameters, such as the available budget, and so on.

- Classification analysis is often used by companies to detect spam, identify possible correlations between potential customers, as well as manage user classifications before and after certain senior events (e.g. advertising campaigns).

- Anomaly detection, to detect inconsistencies or anomalies, this methodology is widely used in both large companies and SMEs. It allows identifying anomalies, malfunctions, or inconsistencies concerning the database management and the data itself. They are often translated into machine learning and knowledge discovery algorithms.

- Intrusion detection, a methodology widely used for the management of intrusion detection problems, with particular attention to the problems of contamination of networks and companies databases.

- Association rule learning is widely used in the sales activities managed by the various companies to identify and manage the relationships between data, 
particularly interesting for the marketing campaigns. Association rules techniques and association mining are examples of these methodologies.

- Decision trees are methodologies used for the optimization of project risk management; they are often used in cost/benefit analysis activities, widely used in risk analysis activities, and in the analysis of activities to be undertaken, schematized through tree graphs.

- Neural networks to automate learning, these methodologies allow to define learning algorithms, mainly related to the management of company data, and activities to be performed under certain conditions (pattern learning), to achieve certain objectives both within the company and aimed at the market and business activities.

- Rule induction includes methodologies developed to perform analysis on data of a company databases and to perform forecasting activities that will be carried out according to defined conditions. Examples of these situations are represented by all the activities related to the management of material purchases or product sales according to the markets to which a specific company is addressed.

- Finally, data warehousing for the big data section is a methodology that particularly concerns the profiling of customers, through the structured aggregation of their information, for the management of information extraction activities, consequent analysis, and decision-making activities in a given economic sector.

It is important to underline that data mining models do not use solutions that are defined better than others are, but each technique refers to certain objectives and data types to be analysed. Moreover, often the best results to transform data into information are obtained through a combination of different analysis techniques. From a general point of view, a data mining process can be summarized by a series of sequential steps, as shown in Figure 3.

Figure 3

Main Steps of a General data mining Process

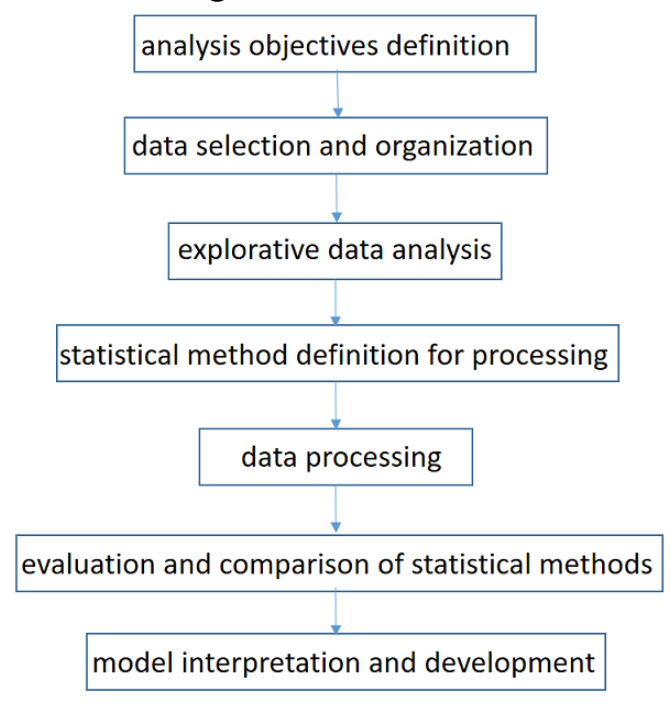

Source: Author's work (2020) 


\section{Data mining and SMEs}

Data mining, considered as a component of the wider knowledge discovery process, allows identifying, in the information stored in a database, models, and relationships hidden in the data (Witten et al., 1999). However, the need to know the activities, to understand the data, or to be aware of general information as well as the statistical methods used remains unchanged.

Nevertheless, as presented in the literature by Rygielski et al. (2002), data mining tools make it possible to answer business questions that in the past took too long to pursue. Literature studies in this field have shown that the answer to these problems that companies still face today is the pivot on which CRM is still based (Thomsen \& Pedersen, 2009; Wanghualin, 2010).

There are different techniques among data mining solutions, each with its advantages and challenges for different types of applications and for different types of companies and markets to which they refer.

\section{Customer relationship management services}

Rygielski et al. (2002) explain how customer relationship management represents a new application area where companies can gain a competitive advantage. Particularly, the application of data mining techniques allows the extraction of hidden predictive information from large databases, as well as the organizations, to identify valuable customers, predict future behaviours, and enable companies to make proactive knowledge-driven decisions.

As specified in the literature, CRM is defined by four main concepts: knowing, destining, selling, and serving (Coomar, 2020; Gurău et al., 2003). The focus then shifts from the product to the customer and the services it can benefit from, shifting market strategies from the outside to the inside. As the authors point out, CRM is based on the analysis conducted by the company towards its customers and the market to which it addresses, to define personalized offers, mainly aimed at customer loyalty, always through a series of services (customer care). Figure 4 reports the main services blocks adopted by a CRM system (as also reported by the literature (Stringfellow et al., 2004, Wanghualin, 2010), by showing the main activities carried out and their correlations.

Figure 4

Customer relationship management main services blocks

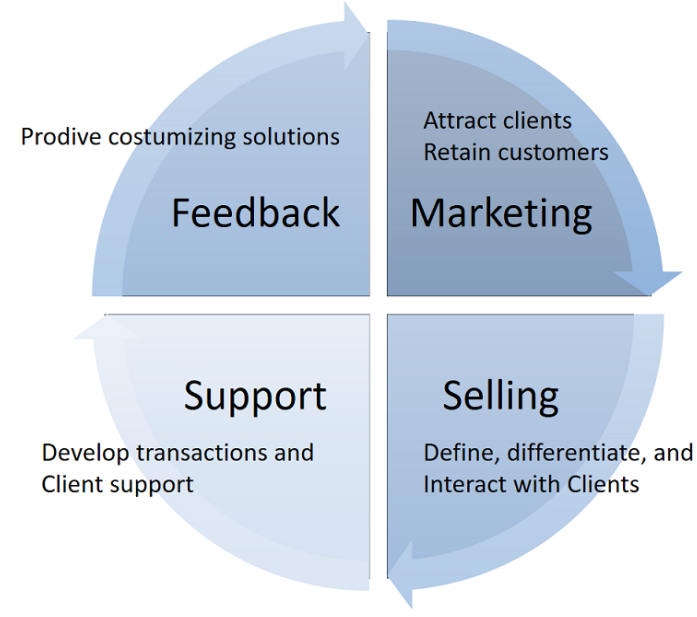

Source: Author's work (2020) 
In this regard, companies mostly push their development of customer orientation by integrating CRM across the entire customer experience chain, leveraging technology to achieve real-time customer management, and constantly innovating their customer value proposition (Linoff \& Berry, 2004). In this sense, data mining techniques are widely used in data analysis, knowledge extraction, and subsequent decision-making activities, based on the most attractive segmentations of the market.

\section{Data mining and customer relationship management solutions in marketing}

Data mining represents, in this field, a tool, widely used by companies, especially SMEs, in the definition and management of customer relations, to adopt increasingly a "customer-oriented" approach. This activity is fundamental for understanding the customer life cycle, because it relates directly to customer revenues and their profitability and because it allows highlighting the weak points of the customercompany relationship, to resolve where possible or reduce critical aspects.

Moreover, the customer's life cycle provides a good framework for the application of data mining, often by giving useful input information related to a specific customer (e.g. on habits), and at the same time by providing useful information on potential customer interests.

Companies report different examples of applications of data mining technologies. The most common in CRM are discovery and predictive modelling activities (Skaates \& Seppänen, 2002). Examples are represented by:

- Retail, including activities of client segmentation through data acquired from store-branded credit cards. Examples of such analysis activities concern performing basket analysis, sales forecasting, handling of the behavioural marketing databases (e.g. cost-effective promotions), merchandise planning.

- Banking, including card marketing analysis, fraud detection applications, and predictive life-cycle management (examples consider data mining solutions that support banks by predicting customer's lifetime value).

- Telecommunication, comprising call detail record analysis, and customer loyalty, where the companies can use data mining to identify the characteristics of customers who are likely to remain loyal once they switch, thus enabling the companies to target their spending on customers who will produce the most profit.

- Different knowledge discovery applications.

Data mining techniques can support high amounts of data, of different nature, such as textual, numerical values, images, audio recordings, etc., by allowing analysis based on a high number of observations and by considering different and numerous variables, that comes from the continuous evolution of the market. These techniques can also be defined through algorithms, developed to address the critical aspects emerging from more traditional solutions, able to minimize, where possible, the computational effort, while ensuring results, even though locally optimal, and simple interpretations by customers. The graphical representation of these advantages is explained in Figure 5. 
Figure 5

Advantages of data mining solutions in the context of SMEs

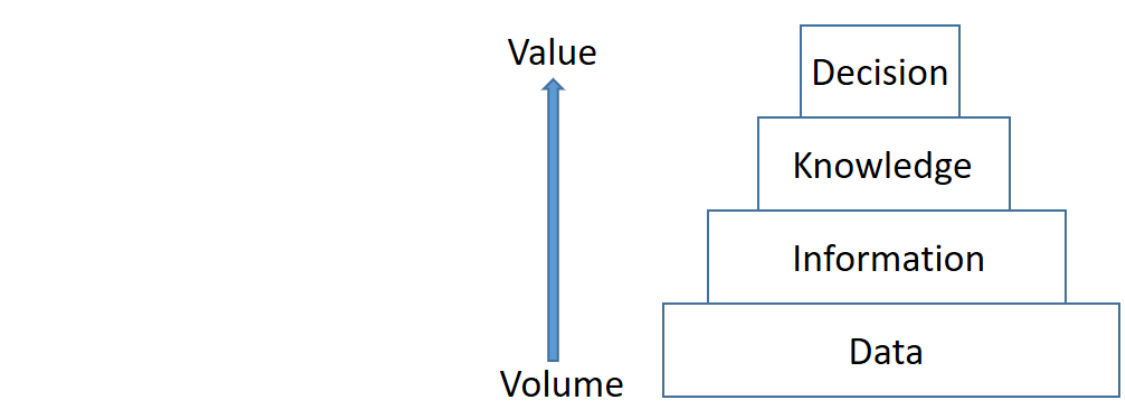

Source: Author's work (2020)

\section{Information technologies and SMEs in Italy}

\section{Background}

In the italian scenario, several companies associations have tried to capture and analyze how information and communication technology is changing the way companies organize their work. Among them the most important and widest survey has been provided by the Italian Association of information and communication technology companies (Assintel), handled by the International Data Corporation (IDC) (IDC, 2020). Their 2019 Report (Assintel, 2019) describes a wide and rich analysis of the actual Italian situation and it has been taken as a source of most of the numbers and concepts described.

A picture of the recent Italian situation highlighted by the Assintel report (Assintel, 2019) shows, on the one hand, a growth of the information technology market, with particular attention to the software and information technology services area, while on the other hand a decrease in investments in the telecommunications services market. According to experts, compared to the hardware market, software confirms that it is the main driver of growth in information technology spending in Italy, with an increase of $+4.7 \%$ in 2018 and $+5 \%$ in 2019 (Assintel, 2019).

The increase is mainly observed in application software, thanks in particular to the growth of investments in the company resource management and relationship management, collaboration, and content management applications (customer relationship management, collaborative, and content applications) markets. These markets have seen an increase in turnover by companies of $+6.5 \%$ and $+8 \%$ respectively over the last two years (Giornale delle PMI, 2019).

An increase in investments was also highlighted by the same report in the context of application development \& deployment. In the latter case, it is above all the applications more closely linked to data management, analysis, and access (data access, analysis \& delivery, and data mining software) that have enabled significant growth to be recorded. This trend, therefore, underlines the importance of data and its management for business success.

Moreover, regarding the objectives that Italian companies are pursuing through the digital transformation initiatives, another interesting focus concerning the CRM emerges in two areas: the improvement of the relationship with customers, in terms of increased customer experience and loyalty, and the improvement of automation levels and internal efficiency. While larger companies primarily pursue automation and internal efficiency objectives, micro companies are aiming in a way priority to improve the relationship with the client, which once again emerges as an area of strong focus by Italian companies. Impacts in the pursuit of this objective are evident in the strategies, processes, and services provided: the digitization initiatives aim, 
indeed, to attract and retain customers, transforming in some cases the way products and services are created and presented, to renew interactions, through the adoption of an increasingly omni-channel approach, and in some cases to enable advanced analysis of customers and their behaviour (customer intelligence, behavioural analysis).

\section{Critical outlook to the digital transformation in Italian SMES}

In agreement with the international trend, also in Italy, we can observe a growth towards a technological evolution that goes in the direction of the so-called "Digital Transformation" phase. There are scenarios in which investments are devoted to implementing solutions more aimed to define and improve knowledge management, such as cloud computing, social business, big data as well as machine learning solutions. This is demonstrated by examples of solutions recently considered in the investment plans of Italian companies, both large and SME, such as the so-called virtual reality augmented reality, robotics, 3D printing, internet of things, currently classified as innovation accelerators, since they represent technologies capable of innovating different processes, knowledge management, data management, and the market in general, showing themselves in a new and efficient way in the economies considered. This shifts the focus of Italian companies' investments from an approach oriented to the maintenance of existing infrastructure to solutions that concretely allow innovation and a more competitive market positioning. Activities such as archiving, knowledge management, and data analysis are the main challenges that also Italian companies have to face today and even more so in the coming years, mainly taking into account the global growth in data volume. It is therefore essential to have solutions capable of dynamically managing data and business knowledge (Corso et al., 2003). Italian companies are also increasingly moving towards the adoption of big data analytics and machine learning solutions, which registered a growth of $17 \%$ in 2019 (Assintel, 2019).

Nevertheless, despite the opportunities of internet of things, there are still few companies in Italy that are developing concrete projects. In fact, according to IDC (International Data Corporation) survey data, only a small percentage of companies are experimenting with internet of things, mainly through feasibility studies and pilot projects. Not surprisingly, micro and small enterprises are still in a phase of study and evaluation of the topic. Large enterprises are the most dynamic in this area and are positioned as those that seem to have a better understanding of the strategic impact of internet of things and are therefore carrying out pilot projects or real concrete activities. Another $14 \%$ of large enterprises are undertaking feasibility studies, followed by $7 \%$ of companies that are carrying out trials and pilot projects. On the other hand, $14 \%$ of large enterprises are in a phase of industrialization of the experiments carried out or are developing a concrete project (Corso et al., 2003). The main driving forces behind the implementation of internet of things projects are the possibility of improved productivity and competitiveness: Italian companies are beginning to become aware that internet of things technologies can help them to compete more effectively, reduce operating costs, increase productivity, innovate the offer and generate new sources of revenue, also reaching new customers and increasing the customer experience.

The sectors that more than others are working on concrete projects in internet of things are retail, mainly in the field of product testing and quality control, and public exercises, tourism, and reception, more oriented to behavioural analysis and projects in the field of customer interaction. Wholesale trade is mainly characterized by being in a phase of industrialization of experimentation and companies in the sector are 
working on the management of the physical security of buildings, plants, and other assets. The Industry sector is in a phase of experimentation and pilot projects and this case, the areas explored are different: security of buildings, plants, and assets, efficient energy management and consumption monitoring, as well as logistics and fleet management, behavioural analysis, and customer interaction. The impacts of the internet of things in the industry sector are perhaps the most evident today and the National Enterprise Plan 4.0, is helping to encourage companies to invest in this type of solutions, which allow a renewal of production facilities, the convergence between physical and virtual production spaces, real-time monitoring of activities and the collection of strategic data directly from the field, for advanced analysis of strategic support to decision-making processes. In many cases, companies in the Industry sector are beginning to give internet of things strategic importance that can help them to compete more effectively in markets undergoing a profound transformation, through the launch of innovative products and services, and to reduce operating costs in some cases. Other sectors, such as public administration, health and education, and finance, are still in a feasibility study phase or have started some internet of things pilot projects. In these cases, we are mainly exploring the opportunities offered by the Internet of Things in the areas of building and plant security, energy management and consumption monitoring, and identification of staff and company staff.

Despite the experiments, pilot projects and some examples of concrete applications, there are still several obstacles to the full explosion of internet of things within the digitization strategies of Italian companies and among these, there are concerns about data security, privacy, and problems related to their management and analysis: in fact, it seems not yet clear how to extract real value from the amount of data collected through internet of things systems and applications to generate new business opportunities and enrich the products and services offered to customers. The other obstacles relate to a general lack of adequate economic resources to be allocated to investments in this area, in addition to security concerns: in this case, there are still several uncertainties regarding the most appropriate measures and technologies that companies should implement for the protection of hardware, software, and networks that underpin internet of things projects.

The perception of the role of information technology security has always been particularly problematic in Italy. The development of a specific culture requires a radical evolution in the understanding of the information technology risk that companies of all levels and levels face when they connect to a network. Only $19 \%$ of Italian companies consider information technology Security as a strategic investment to enable digital transformation models: there is some progression in the perception of information technology Security compared to the more recent past, but it is still a rather slow evolution, which mainly concerns medium and large enterprises (where the figure is over $45 \%$ ). In fact, $35 \%$ of Italian companies consider security only as an additional cost item, like many others, within the budget, more and more articulated and complex, necessary for the management of the company information technology, and $32 \%$ consider it as a contingent and occasional cost, an expense to be faced once in a while, and then completely forget about the problem (especially among micro and small enterprises) (Sali, 2020).

The perception of security also characterizes the industrial sectors in distinct polarities: finance is the sector that believes more in the role of information technology security as an enabling technology to progress in the digital transformation field (almost $33 \%$ of the companies), while at the opposite extreme is the public administration, where almost $70 \%$ of the companies believe that security is only a contingent and entirely occasional expense (Assintel, 2019). It should be noted that 
an evolved culture of security is particularly highlighted in the South and Islands despite other geographies (which are very often dominated by an entrepreneurial fabric characterized by small manufacturing companies, not always inclined to this type of expenditure). Consistent with the basic assumption that information technology security does not assume any strategic investment value, not even in a context now widely regulated by new laws and regulations such as the GDPR, the survey conducted by IDC (IDC, 2020) shows that Italian companies allocate only an extremely limited portion of the company's information technology budget to Security technologies: over $42 \%$ of companies dedicate up to $3 \%, 13 \%$ between 3 and 10\%, only $2 \%$ above $10 \%$.

\section{Digital transformation in Italian SMEs supported by data mining}

The progressive diffusion of digital transformation initiatives and projects in Italian companies is helping to outline an evolutionary framework in which both organizational structures and working methods change, as well as the physical environments and spaces within the companies themselves. The issue of Enterprise 4.0, supported also at government level with incentives of various kinds, from funding facilitations to tax relief measures, will contribute shortly to support the growth of companies that innovate their processes and supply system, thus promoting the development and use of digital technologies in a pervasive way. In this context, it will, be necessary to embrace a culture of innovation, providing for diversified research and development and training activities, but also to adopt advanced security policies and tools, capable of protecting systems, data, and information. These, in fact, already today must necessarily be accessible even outside the company perimeter to enable agile work policies and automation and innovation of processes, not only operational but also decision-making and strategic. Digitization is helping to change the way we work and redesign the physical and logical spaces of companies: in the era of Digital Transformation work activities are starting to be less tied to a physical location and rigid hours, but thanks to the possibility of accessing company resources and information from anywhere, the prospects of "agile" work are opening up for employees. In this context, for several years now, some companies and public bodies have been adopting policies that provide for the introduction of new and flexible measures to better reconcile work and private life; these initiatives are also promoted and regulated in many cases at the legislative level.

According to the research conducted by the International Data Corporation (IDC), more than half of the companies surveyed have launched at least one Smart Working initiative (IDC, 2020). The main activities implemented by companies are so far geared towards ensuring the possibility of working remotely ( $26 \%$ of companies) and enabling employees to work with mobile devices (22\%). The sectors that stand out more than others for having adopted policies of this type are finance, wholesale, industry, transport, communications and utilities, and other services. The objectives of the projects and initiatives undertaken so far are aimed at ensuring greater flexibility and efficiency in work activities, with a greater emphasis on objective-based assessment and the possibility of a better balance between work and personal time. Thanks to initiatives of this kind, it is often necessary for companies to redesign physical spaces as well, for example by reducing fixed workstations and expanding shared workspaces, in addition to promoting the use of digital technologies by employees and strengthening systems for measuring and evaluating individual and collective performance. In this way, in addition to making staff more responsible, as the assessment is based more on professional objectives and is not limited to physical 
presence in the office and rigid working hours, greater collaboration and sharing of activities among employees is enabled.

Moreover, following the research team of the Digital Innovation Observatory of the Politecnico of Milan, the Smart Working is defined as "a new management philosophy founded on a return to people being given flexibility and autonomy in choosing their spaces, their working times and the tools they use, against a backdrop of taking more of innovation in work models within a smart working perspective and for developing methodologies based upon a multi-disciplinary approach that can support decisionmakers (information technology managers, ClOs, human resource managers, and facility managers) in public and private companies" (Smart Working Observatory, 2020).

Also by what reported by the same Italian Observatory, Smart Working represents an organizational phenomenon of growing interest in Italy, which is increasingly becoming an object of planning by organizations. The authors also refer that the number of smart workers, workers who enjoy flexibility and autonomy in the choice of time and place of work and who are equipped with digital tools suitable for working on the move, is constantly growing and today there are about 570 thousand. Currently, $24 \%$ of SMEs refer to this mode of work (Smart Working Observatory, 2020).

The Italian Observatory also shows that it is mainly medium-sized and large companies that adopt smart working policies, allowing remote working, and providing employees with the ability to work using mobile devices. Large enterprises - more than others - seem to understand the benefits of such policies (53\% allow remote working) and are oriented towards the creation of digital workspace that tends to converge and integrate all the services that support business operations and processes, including communication and collaboration, as part of the digital evolution of physical work environments.

Soon, the digital workspace paradigm is expected to become more and more widespread, progressively replacing the traditional physical workstation paradigm:, thanks to the increasing use of digital communication platforms based on data mining technologies, as well as sharing tools and mobile terminals and applications, the possibility to work from anywhere, to be recognized and measured by systems by objectives, will be extended, by also promoting dialogue and collaboration between people, innovating processes and operating environments. Examples of these solutions are, even if partially, collected by the literature shows, in a wider context, interesting applications of data mining in for learning systems (Blagojević \& Micić, 2013), internet of things systems (Alam et al., 2016; Lee \& Lee, 2015) and Knowledge Management Systems (Alavi \& Leidner, 2001; Kovalev et al., 2018; Luo, 2008), and more general open-source approaches in (Hsu, 2009; Landset et al., 2015; Thomsen \& Pedersen, 2009).

The above-reported evolutions will also offer companies the opportunity to focus more on the company's activities and business, while also improving employee satisfaction, thanks to greater flexibility to reconcile work and private life. In a context in which the digital revolution will continue to impact the company's organization and markets, transforming them profoundly, collaboration platforms will become an important vehicle to build and spread knowledge among people and to stimulate and govern the circulation of ideas resulting from shared work.

Among the major innovations in the working environment, technologies that allow the automation of tasks and processes, which allow us to perform more or less complex tasks without the support of human beings, stand out: artificial intelligence and cognitive computing systems will represent the major evolution in this area. The topic is still emerging for Italian companies: according to the survey data, about $70 \%$ 
do not foresee any impact on work or, in any case, a rather limited impact, due to the lack of skills and knowledge on the topic, but also for unclear "business cases" and legal issues related to data management. Despite this main orientation, a small percentage of companies (16\%) already foresee positive and extended impacts for automation technologies on their processes to ensure greater efficiency and productivity of work activities. In the short term, the most significant developments in artificial intelligence and cognitive systems will be recorded in the field of customer service automation and conversational platforms, which are based on the analysis and understanding of natural language: these will allow the development of applications and systems capable of interacting, dialoguing and supporting both employees and customers of the company on certain support services, automating repetitive activities of varying complexity.

The evolution of this type of system will be characterized by a greater capacity to adapt to different processes, able to adapt through the formulation of hypotheses, and to improve through continuous interaction with users. Artificial intelligence and cognitive computing systems are also beginning to be used for the automation of security activities (for example, in the threat intelligence and prevention system field) and in production and distribution processes, thanks to applications in predictive maintenance and intelligent automation field. The drivers that will guide the progressive expansion of this type of systems and technologies are the possibility to receive accurate forecasts, based on advanced analytical models and algorithms, strategic recommendations on decisions and actions to be taken to optimize and improve processes and operations, and a better knowledge of customers and new ways of interaction. Despite the positive impacts that artificial intelligence and cognitive computing systems can have in companies, there is a small share of reality that highlights how there are also resistance and critical issues related to these technologies: $13 \%$ of companies that participated in the survey say they perceive negative impacts related to work automation technologies, mainly due to resistance from employees. In this case, we refer to concerns about the possible replacement of human beings in the performance of activities, especially the simplest and most repetitive ones. Another area that is experiencing significant growth among Italian companies is that of connected objects, i.e. the Internet of Things: through the implementation of connected devices and platforms and thanks to the growing development of support applications companies are experimenting with the opportunities that this type of technology can offer, both in the field of innovation and process efficiency and in the innovation of products and services.

Moreover, digital innovation and data mining can become key drivers for the productivity and performance of SMEs, helping them to compete in a market that will be increasingly interconnected and digitally driven. According to the work carried out by researchers of the Digital Innovation Observatory in SMEs of Politecnico di Milan (Sali, 2020), today, there are about 200 thousand SMEs in the Italian market, and although numerically they represent only $5 \%$ of the entrepreneurial fabric, they represent an important slice of the Italian market: they alone generate $41 \%$ of the entire turnover and $38 \%$ of the added value.

In this context, the analysis of the data is also of particular interest to SMEs, which, according to a published contribution of the editorial staff of the Italian SMEs Journal (Giornale delle PMI, 2019) in 62\% of cases have made investments in 2019, mainly focused on the definition and implementation of technological solutions in the integration of internal data $(80 \%)$, basic training on data analysis for resources already present in the company $(66 \%)$, integration of data from external sources $(57 \%)$ and the development of predictive analysis projects (four out of ten, $+10 \%$ ). The main 
objectives of the investments appear, according to the authors, to be the integration of data mining with digital innovation solutions for the optimization of the supply chain, particularly in manufacturing, the analysis of the competitive environment, and the need to increase the effectiveness of marketing campaigns. Among the companies that have implemented such projects, the results are perceived as highly innovative in $29 \%$ of cases. $40 \%$ of the sample developed advanced analysis projects, at least predictive, usually relying on external expertise. $18 \%$ show good maturity in the development of descriptive analysis and integration of internal data and are also working on the integration of external data, as well as showing interest in employee training (six out of ten have implemented data analysis training plans). $4 \%$ is only focusing on investments in internal data integration, while $38 \%$ have not initiated any initiatives or investments and do not perceive the benefits of Analytics projects.

Moving the analysis to skills, the distance from large enterprises is widening: only $16 \%$ of SMEs have at least one Data Scientist and just over one in five (23\%) at least one Data Analyst. Not far away are the numbers of medium-sized enterprises alone, where the Data Analyst is present in one in three companies. In companies that have taken on Data Science profiles, the results of the projects are perceived as very innovative in $40 \%$ of the cases, compared to $21 \%$ of companies that use only external collaborators.

\section{Conclusion}

In this paper an analysis of the impact of digitalization, and in particular, data mining techniques in the context of CRM applied to boost SMEs development is presented. We analyse only open source tools because they are the most used by SMEs. The analysis presents a brief review of the data mining techniques available and shows how they are practically employed in small companies. Then we also present an economical review of investments in data mining projects in Italy, which on one side outlines that, once used, data mining techniques can boost a company in the market, on the other side the awareness of data mining as a company asset is still not strong in Italian SMEs and most investments in Italy are still carried out by biggest companies.

This aspect is underlined by the fact that, basically, Italian companies see data mining and, more generally, machine learning, as a carrier of innovation and optimization inside the organization and outside, to provide new services and products, but they are still very "prudent" in the investments, due to a difficulty to find personnel with the right skills both internally and in the recruitment market. Moreover, the perception of the role of information technology Security has always been particularly problematic in Italy. The development of a specific culture requires a radical evolution in the understanding of the information technology risk that companies of all levels face when they connect to a network. Only $19 \%$ of Italian companies consider information technology Security as a strategic investment to enable Digital Transformation models, and this slows down the rate of Italy changes.

The presented snapshot of the Italian market shows how digital innovation is pushing socio-economic logics to an unstoppable change in Italy, with a transformative approach that requires new personal attitudes, inside processes, inside every single enterprise, in territorial and regional contexts and aggregations, and so on up to the Country System.

Differently, from previous research, this paper aims to outline the contribution, both technological and economical, that data mining provides to the development of the Italian small and medium companies, which are still the core of the Italian market.

This contribution highlights how one of the most important advantages, coming from the application of data mining tools, is based on the possibility of integrating (in 
companies where it is possible) decision-making processes with constructed rules, synthesizing high information assets. This information can be further converted into commercial actions to obtain a business advantage. In this field, data mining represents, then, a tool, widely used by companies, especially SMEs, in the definition and management of customer relations, to adopt increasingly a customer-oriented approach. Consequently, the application of data mining techniques in customer relationship management is worth pursuing in a customer-centric economy.

Interesting possible future works in this direction could concern the analysis of the development of technologies based on data mining in Italian SMEs, with a comparison with the previous situation they occupied in the Italian and international market. Further studies could also interest the new emerging technological solutions in the economic market, which increasingly play a valuable role in the business.

\section{References}

1. Abazi, B. (2016), "An approach to the impact of transformation from the traditional use of information and communication technology to the Internet of Things: How smart solutions can transform SMEs", IFAC-PapersOnLine, Vol. 49 No. 29, pp. 148-151.

2. Alam, F., Mehmood, R., Katib, I., Albeshri, A. (2016), "Analysis of eight data mining algorithms for smarter Internet of Things (IOT)", Procedia Computer Science, Vol. 98, pp. 437-442.

3. Alavi, M., Leidner, D. E. (2001), "Knowledge management and knowledge management systems: conceptual foundations and research issues", MIS Quarterly, Vol. 25 No.1, pp. 107136.

4. Ali, A. B. M. S., Wasimi, S. A. (2009), "Data mining methods and techniques", Thomson Learning Australia. South Melbourne.

5. Almeida, P., Bernardino, J. (2016), "A survey on open source data mining tools for SMEs", In New Advances in Information Systems and Technologies, Springer, Cham, pp. 253-262.

6. Assintel. (2019), "The information and communication technology market and the digital evolution in Italy", Annual report, Assintel, Milano.

7. Berson, A., Smith, S., Thearling, K. (1999), Building Data Mining Applications for Customer Relationship Management", Mc-Graw Hill, New York.

8. Blagojević, M., Micić, I. (2013), "A web-based intelligent report e-learning system using data mining techniques", Computers \& Electrical Engineering, Vol. 39 No.2, pp. 465-474.

9. Calvello, M. (2020), "Learn data mining Techniques", available at: https://learn.g2.com/data-mining-techniques (Apr 27, 2020)

10. Cedrola, E., Battaglia, L., Tzannis, A. (2009), "The Italian SMEs in the International context. A model to succeed in the global arena", Internal Report of the University of Macerata, No. 52. pp. 1-24.

11. Chen, I. J., Popovich, K. (2003), "Understanding customer relationship management (CRM): People, process and technology", Business Process Management Journal, Vol. 9 No. 5, pp. 672-688.

12. Coomar, D. (2020), "Elements of customer relationship management Implementation", available at: https://www.engagebay.com/blog/crm-implementation/ (Apr 27, 2020)

13. Corso, M., Martini, A., Paolucci, E., Pellegrini, L. (2003), "Knowledge management configurations in Italian small-†o-medium enterprises", Integrated Manufacturing Systems, Vol. 14 No. 1, pp. 46-56.

14. Fayyad, U. M. Piatetsky-Shapiro, G., Smyth, P. (1996), "From data mining to knowledge discovery: An overview", in Advances in Knowledge Discovery and Data Mining, American Association for Artificial Intelligence, Menlo Park, pp. 1-34.

15. Ghaderi, H., Fei, J. (2013), "Data mining practice in SMEs: A customer relationship management perspective", in Lane, R., Kahn, D. (Eds.), 11 th ANZAM Operations, Supply Chain and Services Management Symposium: Research Industry, 20-21 June, Australian and New Zealand Academy of Management, Brisbane, pp.1-12. 
16. Giornale delle PMI. (2019), "The Data Analytics market in Italy", available at: https://www.giornaledellepmi.it/il-mercato-dei-big-data-analytics-in-italia-vale-17miliardi-di-euro-23/ (Apr 27, 2020)

17. Gurău, C., Ranchhod, A., Hackney, R. (2003), "Customer-centric strategic planning: Integrating customer relationship management in online business systems", Information Technology \& Management, Vol. 4 No.2, pp. 199-214.

18. Hsu, C. H. (2009), "Data mining to improve industrial standards and enhance production and marketing: An empirical study in apparel industry", Expert Systems With Applications, Vol. 36 No. 3, pp. 4185-4191.

19. Hui, S. C., Jha, G. (2000), "Data mining for customer service support", Information \& Management, Vol. 38 No. 1, pp. 1-13.

20. IDC. (2020), "IDC analyze the future", available at: www.idc.com (Apr 27, 2020)

21. Iqbal, M., Kazmi, S. H. A., Manzoor, A., Soomrani, A. R., Butt, S. H., Shaikh, K. A. (2018), "A study of big data for business growth in SMEs: Opportunities \& challenges", in International Conference on Computing, Mathematics and Engineering Technologies, 3-4 March, IEEE, Sukkur, pp. 1-7.

22. Jun, C., Lee, J. Y., Yoon, J.-S., Kim, B. H. (2017), "Applications' integration and operation platform to support smart manufacturing by small and medium-sized enterprises", Procedia Manufacturing, Vol. 11, pp. 1950-1957.

23. Kovalev, D., Shanin, I., Stupnikov, S., Zakharov, V. (2018), "Data mining methods and techniques for fault detection and predictive maintenance in housing and utility infrastructure", in International Conference on Engineering Technologies and Computer Science, 20-21 March, IEEE, Moscow, pp. 47-52.

24. Landset, S., Khoshgoftaar, T. M., Richter, A. N., Hasanin, T. (2015), "A survey of open source tools for machine learning with big data in the Hadoop ecosystem" Journal of Big Data, Vol. 2 No.1, pp. 1-24.

25. Lee, I., Lee, K. (2015), "The Internet of Things (IOT): Applications, investments, and challenges for enterprises", Business Horizons, Vol. 58 No. 4, pp. 431-440.

26. Linoff, G. S., Berry, M. J. A. (2004), Data Mining Techniques: For Marketing, Sales, and Customer Relationship Management (2nd ed.), Wiley Publishing, Indianapolis.

27. Luo, Q. (2008), "Advancing knowledge discovery and data mining", in First International Workshop on Knowledge Discovery and Data Mining, 23-24 January, IEEE, Adelaide, pp. 3-5.

28. Marbán, O., Menasalvas, E., Fernández-Baizán, C. (2008), "A cost model to estimate the effort of data mining projects CoMo)", Information Systems, Vol. 33 No.1, pp. 133-150.

29. Marzo, G., Scarpino, E. (2016), "Exploring intellectual capital management in SMEs: An indepth Italian case study", Journal of Intellectual Capital, Vol. 17 No. 1, pp. 27-51.

30. Ministry of Economic Development. (2017), "The Italian legislation in support of innovative SMEs Executive summary", DG for Industrial Policy, Competitiveness and SMEs, available at:

https://www.mise.gov.it/images/stories/documenti/executive_summary_innovative_SMEs _23febbraio2017.pdf (Apr 27, 2020)

31. Mourtzis, D., Vlachou, E., Milas, N. (2016), "Industrial big data as a result of lot adoption in manufacturing", Procedia CIRP, Vol. 55, pp. 290-295.

32. Ngai, E. W. T., Xiu, L., Chau, D. C. K. (2009), "Review: Application of data mining techniques in customer relationship management: A literature review and classification", Expert Systems With Applications, Vol. 36 No.2, pp. 2592-2602.

33. Packianather, M. S., Davies, A., Harraden, S., Soman, S., White, J. (2017), "Data mining techniques applied to a manufacturing SME", Procedia CIRP, Vol. 62, pp. 123-128.

34. Pejić Bach, M. (2014), "Exploring information and communications technology adoption in enterprises and its impact on innovation performance of European countries", Ekonomický časopis, Vol. 62, No. 4, pp. 335-362.

35. Rojas-Torres, D., Kshetri, N. (2019), "Big data solutions for micro-, small-, and medium-sized enterprises in developing countries", IT Professional, Vol. 21 No. 5, pp. 67-70.

36. Rygielski, C., Wang, J., Yen, D. (2002), "Data mining techniques for customer relationship management", Technology in Society, Vol. 24, pp. 483-502. 
37. Sali, G. (2020), "Network digital 360: Digital innovation observatory", available at: https://www.digital4.biz/pmi/pmi-digitale-cresce-attenzione-innovazione-a-rilento/ (Apr 27, 2020)

38. Sen, D., Ozturk, M., Vayvay, O. (2016), "An overview of big data for growth in SMEs" Procedia - Social and Behavioral Sciences, Vol. 235, pp. 159-167.

39. Shaw, M. J., Subramaniam, C., Tan, G. W., Welge, M. E. (2001), "Knowledge management and data mining for marketing", Decision Support Systems, Vol. 31 No.1, pp. 127-137.

40. Skaates, M. A., Seppänen, V. (2002), "Managing relationship-driven competence dynamics in professional service organisations", European Management Journal, Vol. 20 No. 4, pp. 430-437.

41. Smart Working Observatory. (2020), "Digital innovation research: The smart working", available at: https://www.osservatori.net/ (Apr 27, 2020)

42. Stringfellow, A., Nie, W., Bowen, D. E. (2004), "Customer relationship management: Profiting from understanding customer needs", Business Horizons, Vol. 47 No. 5, pp. 45-52.

43. Thomsen, C., Pedersen, T. B. (2009), "A survey of open source tools for business intelligence", International Journal of Data Warehousing and Mining, Vol. 5 No. 3, pp. 56-75.

44. Wanghualin. (2010), "Data mining and Its Applications in CRM", in International Conference on Computer Research and Development, 7-10 May, IEEE, Kuala Lumpur, pp. 822-825.

45. Witten, I. H., Frank, E., Hall, M. A. (1999), Data Mining: Practical Machine Learning Tools and Techniques, Morgan Kauffmann, San Francisco.

46. Yang, J., Niu, Z., Wang, S., Li, F. (2011), "Individual customer management system for small and medium enterprises and management method of management system", available at: https://patents.google.com/patent/CN102024202A/en (Apr 27, 2020)

\section{About the authors}

Amir Topalović received his Master Degree in Finance and Risk Management at the University of Parma and he is actually a PhD student in Business and Administration at the University of Sarajevo under the supervision of Prof. Nijaz Bajgoric. His thesis title is "A TOE based Study on Data Mining employment in Italian Small and Medium Enterprises". Currently, he is CEO at C2T, Consortium for the Technology Transfer, and his research interests are related to data mining in corporate and banking data. The author can be contacted at amir.topalovic@consorzioc2t.it

Antonia Azzini is an ICT researcher and innovation manager at Consortium for Tehcnolgy Transfer C2T. With a PhD in Computer Science at the University of Milan, and a long experience as PostDoc at the Computer Science Department of the University of Milan, she is responsible of the cooperation with universities and other research centres. She is member of the Editorial Board of IJKL Journal, and she is a member of the IFIP 2.6 Working Group on Databases. The author can be contacted at antonia.azzini@consorzioc2t.it. 